\title{
Primary Endobronchial Leiomyoma
}

M Unsal $^{1}$, B Celik ${ }^{2}$, Y Sullu ${ }^{3}$, M Elmali $^{4}$, G Kocak

\begin{abstract}
Leiomyoma accounts for less than $2 \%$ of the benign tumours of the lung. These lesions are usually asymptomatic. However, they can present symptoms depending on the localization. The HRCT of a 57-year-old male patient with cough, sputum and shortness of breath ongoing for 2 months revealed a $5 \mathrm{~mm}$ lesion in the inferior lingular bronchus. The report of the bronchoscopic biopsy was leiomyoma, and the patient underwent lingulectomy.
\end{abstract}

Keywords: Laboratory, lung cancer, metastasis, survival, symptoms

From: ${ }^{1}$ Department of Chest Disease, ${ }^{2}$ Department of Thoracic Surgery, ${ }^{3}$ Department of Pathology, and ${ }^{4}$ Department of Radiology, Ondokuz Mayis University Medical School, Samsun, Turkey.

Correspondence: Dr M Unsal, Department of Respiratory Medicine, Faculty of Medicine, University of Ondokuz Mayis, 55139 Kurupelit, Samsun, Turkey. Fax: +90 362 4576070, e-mail:meftununsal@gmail.com 


\section{INTRODUCTION}

Pulmonary leiomyoma, which originates from the smooth muscles in the bronchi, bronchioles, bronchial arterioles or interstitial tissues, is among the rare benign tumours of the lungs. Its prevalence is less than $2 \%$ among all benign tumors of the lung. Half of the leiomyomas reported in the literature is in the lung parenchyma, one-third is in the bronchioles and the rest are in the trachea (1-3). It is often encountered in the $3^{\text {rd }}$ and $5^{\text {th }}$ decades, and its prevalence is 1.5:1 higher in females than males. These asymptomatic rare tumours are often detected incidentally during radiological evaluation. Radiologically; they can be observed as postobstructive pneumonia, atelectasis, endobronchial nodules and intrabronchial or extra-bronchial masses $(2,4)$.

In this article, a primary endobronchial leiomyoma case detected bronchoscopically and treated surgically is presented together with the information in the literature.

\section{CASE REPORT}

The 57-year-old male patient presented to the Clinic of Pulmonary Diseases with the complaints of cough, sputum and shortness of breath which started 2 months previously. In his medical history, the patient had been receiving medical treatment due to bronchial asthma for about 2 years, and that he had been treated as an inpatient in another clinic 2 months before his admittance to our centre. The patient who had smoked 40 pack/year quitted smoking 3 years previously. Rhonchus was heard in the bilateral lower zones during the respiratory system examination. Vital signs and the physical examination of the other systems were normal.

Laboratory findings were haemoglobin $12.6 \mathrm{~g} / \mathrm{dL}$, leukocyte $7590 \mathrm{bin} / \mathrm{uL}$ (PMNL $64.9 \%$, lymphocyte $25.2 \%$, eosinophil $0.6 \%$, monocyte $6.5 \%$ ), platelet $250000 / \mathrm{uL}$, 
erythrocyte sedimentation rate $18 \mathrm{~mm} / \mathrm{h}$ and CRP $16 \mathrm{mg} / \mathrm{L}$. Other biochemical parameters were normal. In the pulmonary function test, FVC was $2.42(61.9 \%)$, FEV1 was $1.90(60.0 \%)$ and $\mathrm{FEV} 1 / \mathrm{FVC}$ was $97.4 \%$.

Posteroanterior chest radiograph of the patient was considered to be normal. (Figure 1A) In high resolution computed tomography, a nodular image $5 \mathrm{~mm}$ in diameter was observed in the inferior lingular bronchus. (Figure 1B)

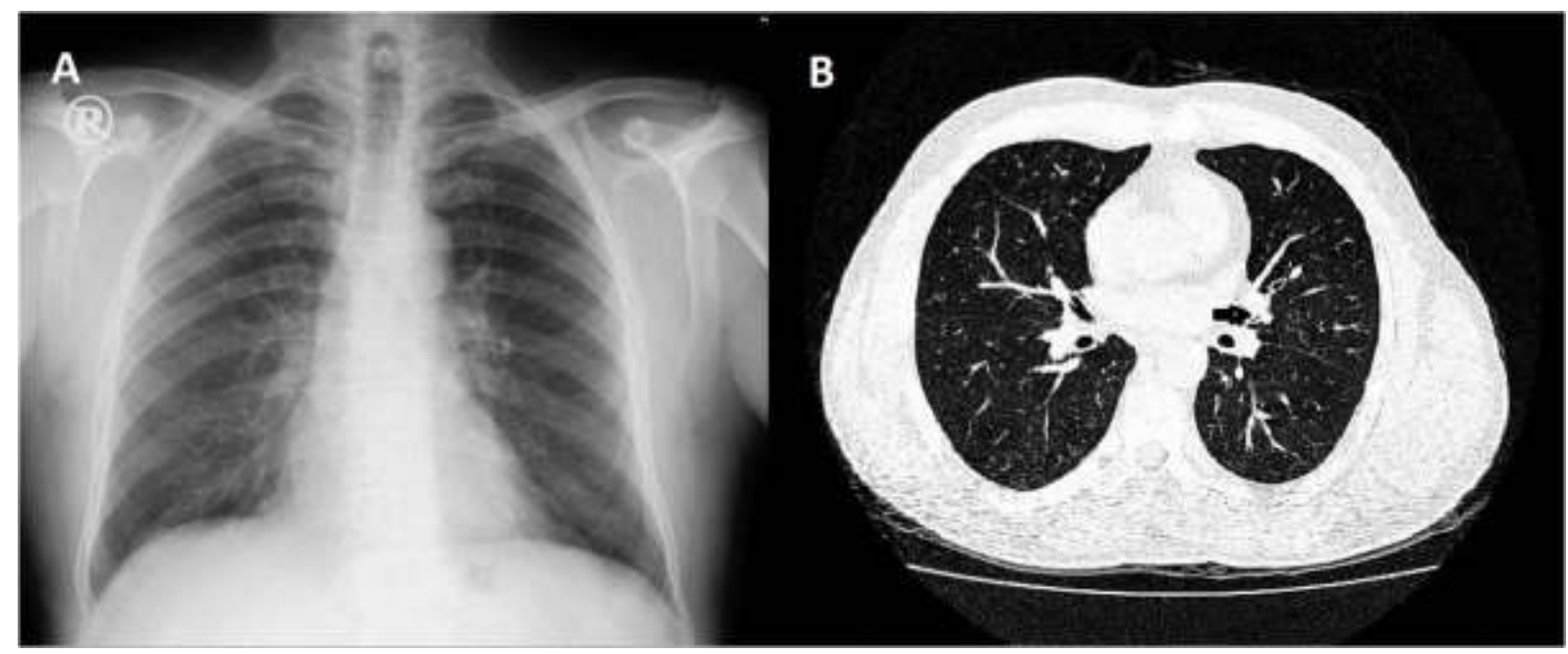

Fig 1: A; chest X-ray is normal, B; thorax $\mathrm{CT}$ revealed a nodul in the inferior lingular bronchus (black arrow).

Fiberoptic bronchoscopy revealed a white, smooth-surfaced, wide-based endobronchial lesion which totally obstructed the entry of the inferior lingular bronchus in the left lung. (Fig. 2) 

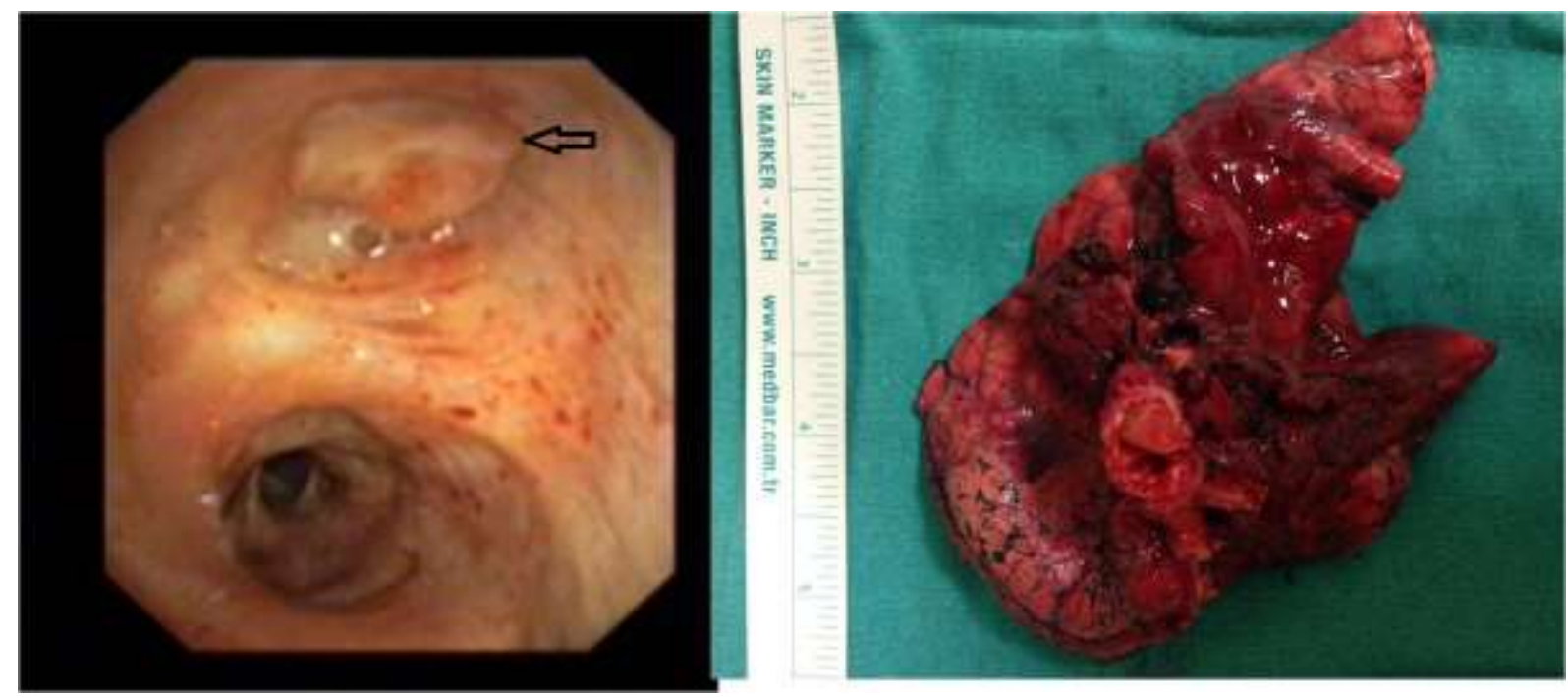

Fig 2: Fiberoptic bronchoscopy revealed a white, wide-based endobronchial lesion in the inferior lingular bronchus (black arrow). We can see the endobronchial lesion in the lingulectomy specimen.

Bronchoscopic biopsy result was consistent with hamartoma or leiomyoma, so surgical treatment was planned. The patient underwent lingulectomy throughout left muscle sparing thoracotomy, and he was discharged on the postoperative $7^{\text {th }}$ day. Histopathologic examination revealed that immediately beneath the pseudostratified ciliated columnar epithelium the tumor was composed of bland spindl cells arranged in whorls and fascicles. Tumor was stained positive for smooth muscle actin and desmin. No atypia, mitoses or necrosis was seen. On the basis of morphological and immunohistochemical findings the lesion was diagnosed as bronchial leiomyoma. (Fig. 3) 


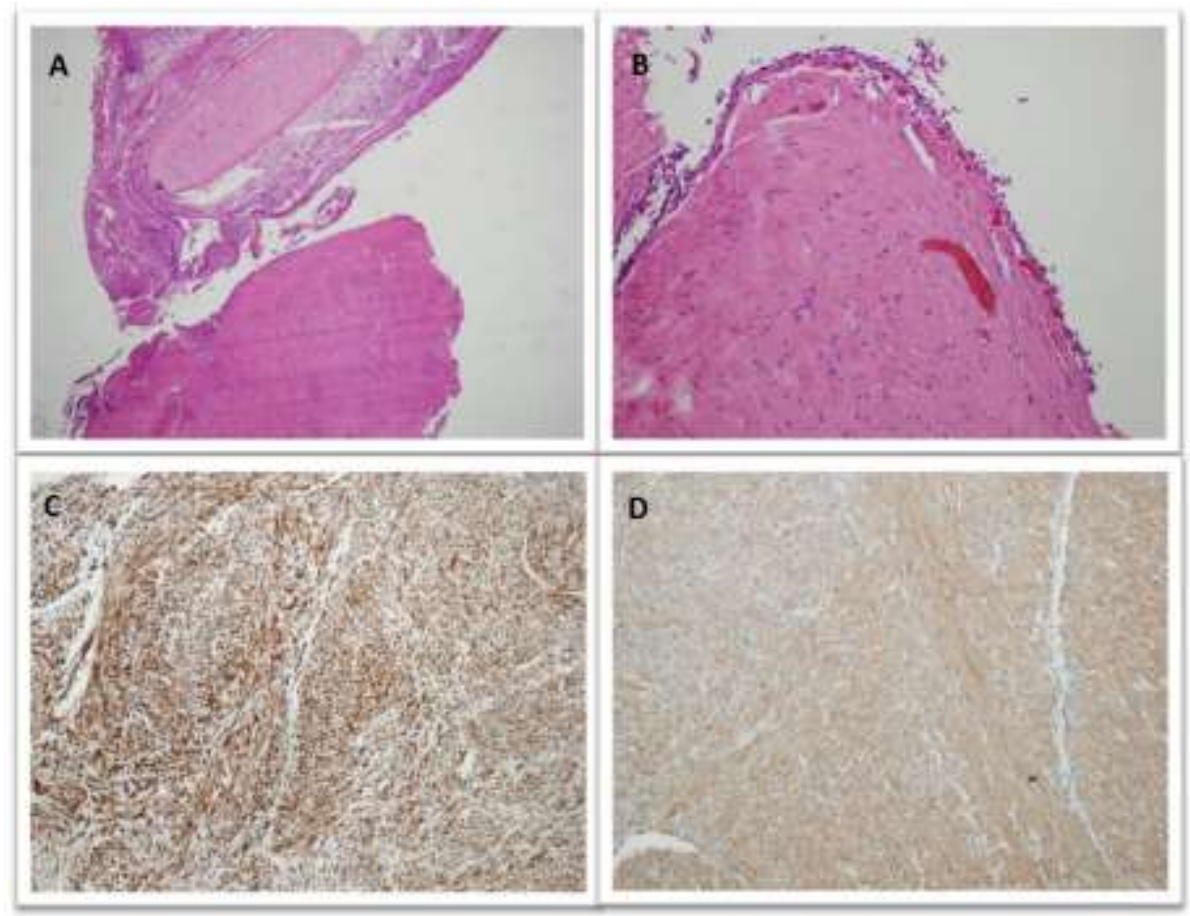

Fig 3: Tumor composed of cells arranged in whorls and fascicles with spindle shaped cells with monomorphous fusiform nuclei. No necrosis or mitotic activity. It showed diffuse cytoplasmic reactivity for smooth muscle actin and desmin. A; H-E x 100, B; H-E x 200; C; desmin x 200, D; SMA x 200

The patient is in the $7^{\text {th }}$ postoperative month now, and no complications have been observed during the follow-up.

\section{DISCUSSION}

Leiomyoma, which was first described by Forkel in 1910, is considered among the rare benign tumors of the lung (5). Patients are usually asymptomatic, but they can present symptoms depending on the localization of the tumour in the lung. If the lesion is localized in the trachea, asphyxia can be observed due to the upper airway obstruction. Endobronchial lesions or the lesions obstructing the bronchus externally can cause haemoptysis, atelectasis, 
consolidation, bronchiectasis or postobstructive pneumonia (2). Since they are benign, develop slowly and cause nonspecific symptoms, they are often diagnosed late.

In radiological evaluation; lesions are often missed on chest radiographs. Thorax CT can reveal the localization and the size of the lesion, its relation with the neighbouring tissues and whether it is pedunculate or not. Bronchoscopy is essential for definitive diagnosis (2). In differential diagnosis, hamartoma, extrauterine leiomyoma/leiomyosarcoma, inflammatory myofibroblastic tumour, inflammatory polyp and carcinoid tumors should be considered (2).

The treatment of pulmonary leiomyoma depends on the localization and the size of the lesion, pedunculate or not and the destruction of the lung parenchyma at the distal of the lesion. Definitive treatment is the surgical or bronchoscopic excision of the lesion. Such techniques as Nd-YAG laser, argon plasma, cryotherapy, electrocautery or snare are used in bronchoscopic methods. Bronchoscopic excision could be performed for the pedunculated lesions. They can be located in the trachea, main bronchi or lobar bronchi. However, complete bronchoscopic excision may not be possible for the lesions seated on a wide base and at the far distal of the lobar bronchi. Furthermore, bronchoscopic excision will not be the appropriate choice if the lung parenchyma at the distal of the lesion is damaged. In such cases, surgical resection should be performed. Lobectomy or segmentectomy should be performed in a way that the lung parenchyma could be spared as much as possible (1-3).

Prognosis depends on the complete excision of the lesion. The prognosis is perfect in complete resection. However, in incomplete resection, local recurrence is common, and patients should be followed periodically (2). In their series of bronchoscopic resection, Kwon et al. performed an incomplete resection, then a surgical resection due to recurrence in 2 patients with wide-based leiomyoma(3) Park et al. performed 8 surgical resections in their series of 16 patients(1). Dmello et al. reported in their article that only $4 \%$ of the pulmonary leiomyoma cases in the literature were localized in the lingual (6). 
Our patient had had the complaints for about 2 years and had been treated and followed up with the diagnosis of bronchial asthma. In his HRCT, a $5 \mathrm{~mm}$ lesion was observed in the entry of the left lung inferior lingular bronchus, and bronchoscopy was performed. Lesion was detected through a careful radiological evaluation and the diagnosis was made. Since the lesion was seated on a wide base, bronchoscopic excision was not considered, and the patient underwent left lingulectomy. Postoperative histopathological evaluation revealed that a complete resection had been performed. The patient is being followed up.

In conclusion, endobronchial leiomyoma is a rare benign tumour of the lung. The treatment method is the complete removal of the lesion. The choice of treatment changes depending on the localization and size of the lesion and pedunculated or not.

\section{ACKNOWLEDGEMENTS}

This study was presented as a poster presentation at 17 th International Congress of the Turkish Thoracic Society, 2-6 April 2014, Antalya, Turkey. We also thank Salih Yilmaz for editing the English in an earlier version of this manuscript.

\section{AUTHORS’ NOTE}

The authors declare that they have no conflict of interest. 


\section{REFERENCES}

1. Park JS, Lee M, Kim HK, Choi YS, Kim K, Kim J, et al. Primary leiomyoma of the trachea, bronchus, and pulmonary parenchyma: a single institutional experience. Eur J Cardiothorac Surg 2012; 41: 41-5.

2. Percinel S, Heper AO, Savas B, Ceyhan K, Enön S, Güngör A. Bronchial leiomyoma with presumptive diagnosis of lung carcinoma: a case report and review of the literature. Turkish Respir J 2008; 9: 41-5.

3. Kwon YS, Kim H, Koh WJ, Suh GY, Chung MP, Kwon OJ, Han J. Clinical characteristics and efficacy of bronchoscopic intervention for tracheobronchial leiomyoma. Respirology 2008; 13: 908-12.

4. Kim YK, Kim H, Lee KS, Han J, Yi CA, Kim J, et al. Airway leiomyoma: imaging findings and histopathologic comparisons in 13 patients. AJR Am J Roentgenol 2007; 189: 393-9.

5. Orlowski TM, Stasiak K, Kolodziej J. Leiomyoma of the lung. J Thorac Cardiovasc Surg 1978; 76: 257-61.

6. Dmello D, Javed A, Espiritu J, Matuschak GM. Endobronchial leiomyoma: case report and literature review. J Bronchology Interv Pulmonol 2009; 16: 49-51. 